\title{
A Meta-Analysis of GBA-Related Clinical Symptoms in Parkinson's Disease
}

\author{
Yuan Zhang $\mathbb{D}^{1}{ }^{1}$ Li Shu $\mathbb{D}^{1},{ }^{1}$ Xun Zhou, ${ }^{1}$ Hongxu Pan, ${ }^{1}$ Qian Xu, ${ }^{1,2,3}$ Jifeng Guo $\mathbb{D}^{1,2,3,4,5,6}$ \\ Beisha Tang $\mathbb{D},{ }^{1,2,3,4,5,6,7,8}$ and Qiying Sun $\mathbb{D}^{2,3,7}$ \\ ${ }^{1}$ Department of Neurology, Xiangya Hospital, Central South University, Changsha, Hunan 410008, China \\ ${ }^{2}$ National Clinical Research Center for Geriatric Disorders, Changsha, Hunan 410078, China \\ ${ }^{3}$ Key Laboratory of Hunan Province in Neurodegenerative Disorders, Central South University, Changsha, Hunan 410008, China \\ ${ }^{4}$ Parkinson's Disease Center of Beijing Institute for Brain Disorders, Beijing 100069, China \\ ${ }^{5}$ Collaborative Innovation Center for Brain Science, Shanghai 200032, China \\ ${ }^{6}$ Collaborative Innovation Center for Genetics and Development, Shanghai 200438, China \\ ${ }^{7}$ Department of Geriatrics, Xiangya Hospital, Central South University, Changsha, Hunan 410008, China \\ ${ }^{8}$ Center for Medical Genetics, School of Life Sciences, Central South University, Changsha, Hunan 410008, China
}

Correspondence should be addressed to Qiying Sun; sunqiying2015@163.com

Received 28 April 2018; Revised 4 August 2018; Accepted 14 August 2018; Published 27 September 2018

Academic Editor: Jan Aasly

Copyright ( $\odot 2018$ Yuan Zhang et al. This is an open access article distributed under the Creative Commons Attribution License, which permits unrestricted use, distribution, and reproduction in any medium, provided the original work is properly cited.

Background. GBA gene had been proved to be a crucial gene to the risk of PD. Numerous studies had discussed about the unique clinical characteristics of PD patients with GBA carriers (GBA+PD). However, there was lack of updated comprehensive analysis on the topic. In order to clarify the association between GBA variants and the clinical phenotypes of PD, we conducted this comprehensive meta-analysis. Method. Medline, Embase, and Cochrane were used to perform the searching. Strict selection criteria were followed in screening for new published articles or data. Revman 5.3 software was applied to perform the total statistical analysis, and funnel plots in the software were used to assess the publication biases. Results. A total of 26 articles including $931 G B A+$ PD and $14861 G B A$ noncarriers of PD (GBA - PD) were involved in the final meta-analysis, and 14 of them were either newly added publications or related data newly analyzed compared with the version published in 2015 . Then, a series of symptoms containing depression, orthostatic hypotension, motor fluctuation, wearing-off, and freezing were newly analyzed due to more articles eligible. Besides, clinical features like family history, AAO, UPDRS-III, H-Y, and dementia previously analyzed were updated with new data added. Significant statistical differences were found in wearing-off, family history, AAO, UPDRS-III, and dementia (OR: 1.14, 1.65; MD: -3.61, 2.17; OR: 2.44; p: 0.03, <0.00001, <0.00001, 0.003, and <0.00001). Depression was slightly associated with $G B A+\mathrm{PD}(\mathrm{OR}: 1.47 ; \mathrm{p}$ : 0.04$)$. Clinical symptoms such as $\mathrm{H}-\mathrm{Y}$, orthostatic hypotension, motor fluctuation, and freezing did not feature GBA + PD. Conclusion. Our results demonstrated that there were unique clinical features in $G B A+\mathrm{PD}$ which can help the management of the whole duration of PD patients.

\section{Introduction}

Parkinson's disease (PD), a common neurodegenerative disease, was featured by motor symptoms containing bradykinesia, resting tremor, rigidity, and postural instability. Nonmotor symptoms (NMS) such as cognitive impairment, olfactory dysfunction, and depression were also common in PD patients. Nowadays, the pathogenesis of PD remains elusive. Genetic factors have been demonstrated to cause PD and, to some extent, participate in modifying the phenotypes of PD [1-3].

GBA gene, encoding the lysosomal enzyme glucocerebrosidase (GCase), is the causative gene of Gaucher's disease (GD) [4]. GBA variants can increase the risk of PD up to 10 times, which was the strongest genetic factor contributing to the risk of PD [5, 6]. Nowadays, more than 300 mutations in GBA were reported [7-9]. The latest comprehensive metaanalysis had proved the importance of $G B A$ variants such as 
L444P, N370S, R120W, IVS2 + 1G > A, H255Q, D409H, RecNciI, E326K, and T369M to PD risks [10].

In addition to the contribution of $G B A$ to the development of PD, studies have reported PD patients with $G B A$ carriers $(G B A+P D)$ manifested special clinical features compared to idiopathic PD. In the year of 2015, our group conducted a study combining the results of our new original research and meta-analysis on the association between $G B A$ variants and the clinical features of PD [11]. The data indicated that $G B A+\mathrm{PD}$ are more inclined to onset at early age, initially with bradykinesia, have family history and develop to dementia when compared with $G B A$ noncarriers of PD patients (GBA-PD). However, with more published articles, there were new clinical features such as depression, motor complications, and freezing gaits focused by researchers which will help draw a full picture of clinical features of $G B A+\mathrm{PD}$ or $G B A-\mathrm{PD}$ with complete motor symptoms (MSs) and NMSs [12-14]. Combined with newly published articles and newly involved data of previous articles, we performed a comprehensive analysis on clinical features of $G B A+P D$.

\section{Methods}

2.1. Selection Criteria. We conducted this meta-analysis based on PICOS (participants, interventions, controls, outcomes, and studies) rules.

2.1.1. Participants. All PD patients being diagnosed with widely accepted diagnostic criteria [15].

2.1.2. Interventions. DNAs were expanded by PCR-based methods or other accepted methods and analyzed by Sanger sequencing or other regular methods.

\subsubsection{Controls. Controls were PD patients without carrying} $G B A$ variants.

2.1.4. Outcomes. A specific clinical feature of $G B A$ carriers and noncarriers in PD patients were reported.

2.1.5. Study Types. Original studies such as case-only study, cohort study, or case-control study were conducted.

2.2. Literature Search. We searched articles in English using Medline database in Pubmed, Embase database in Ovid, and the Cochrane database. Key words were "GBA," "glucocerebrosidase," "Parkinso*" and "PD." The latest search was done on March 1, 2018. Overlapping articles from different databases were excluded. Two researchers (Yuan Zhang and Li Shu) performed the search independently. In case of disagreements, a third researcher (Qiying Sun) was consulted to arrive at a consensus.

2.3. Data Extraction. Comprehensive data were retrieved including the following items: publication year, first author, ethnicity, country, number of $G B A+\mathrm{PD}$ and $G B A-\mathrm{PD}$, and corresponding clinical information. Two researchers did the extraction independently. Another author was asked to participate in the process when confronted with problems. The quality of all case-control studies were assessed according to the Newcastle-Ottawa Scale (NOS) [16].

2.4. Statistical Analysis. The total statistical analysis was performed in Revman 5.3 software. The final results were demonstrated by pooled odds ratio (OR) or mean difference (MD) and 95\% CI (confidence interval). When the data were dichotomous variables, pooled odds ratio (OR) was calculated, otherwise when the data were continuous outcomes, pooled mean difference (MD) was expressed. Heterogeneity was reflected by $Q$ statistic ( $p$ value) and $I^{2}$ statistic. $p>0.1$, $I^{2} \leq 50 \%$ indicated that the heterogeneity was not significant and suggested a fixed model (FM) be applied. Otherwise, a random model (RM) was used. The shape of funnel plot was used to reflect publication biases. Sensitivity analysis was performed by removing each original study sequentially to test the stability of the results.

\section{Results}

The complete information of searching process is shown in the flowchart (Figure 1), and the information of all included studies is demonstrated in Table 1. A total of 26 articles including $931 G B A+\mathrm{PD}$ and $14861 G B A$ - PD were involved in the final meta-analysis, and 14 of them, which contained $582 G B A+P D$ and $8217 G B A-P D$, were either newly added publications or related data newly analyzed compared with the version published in 2015 [11]. Then, a series of symptoms containing depression, orthostatic hypotension, motor fluctuation, wearing-off, and freezing were newly analyzed due to more articles eligible. Besides, clinical features like family history, age at onset (AAO), Unified Parkinson's Disease Rating Scale Part III (UPDRS-III), Hoehn-Yahr (H-Y), and dementia previously analyzed were updated with new data added. Due to the importance of disease duration in clinical characteristics of disease, we first conducted comparison of disease duration between $G B A+P D$ and $G B A-P D$. We found that there was no statistical difference between the two groups (MD: 0.17, $p$ : 0.47) (Supplementary Table 1; Supplementary Figure 1).

For the five (depression, orthostatic hypotension, motor fluctuation, wearing-off, and freezing) newly involved clinical characteristics in this meta-analysis (Table 2, Supplementary Figure 1), they belonged to NMS and motor complications. As can be seen from Table 2, significant statistical difference was found in wearing-off (OR: $1.14 ; p$ : $0.03)$. Slightly statistical significance was found in depression of GBA + PD (OR: 1.47; p: 0.04). Clinical symptoms such as orthostatic hypotension, motor fluctuation, and freezing did not feature $G B A+\mathrm{PD}$ in this meta-analysis.

As to the five updated clinical features of $G B A+P D$ with newly involved data (Table 2, Supplementary Figure 1), they were family history, AAO, UPDRS-III, H-Y, and dementia. From the tables, significant statistical differences were found 


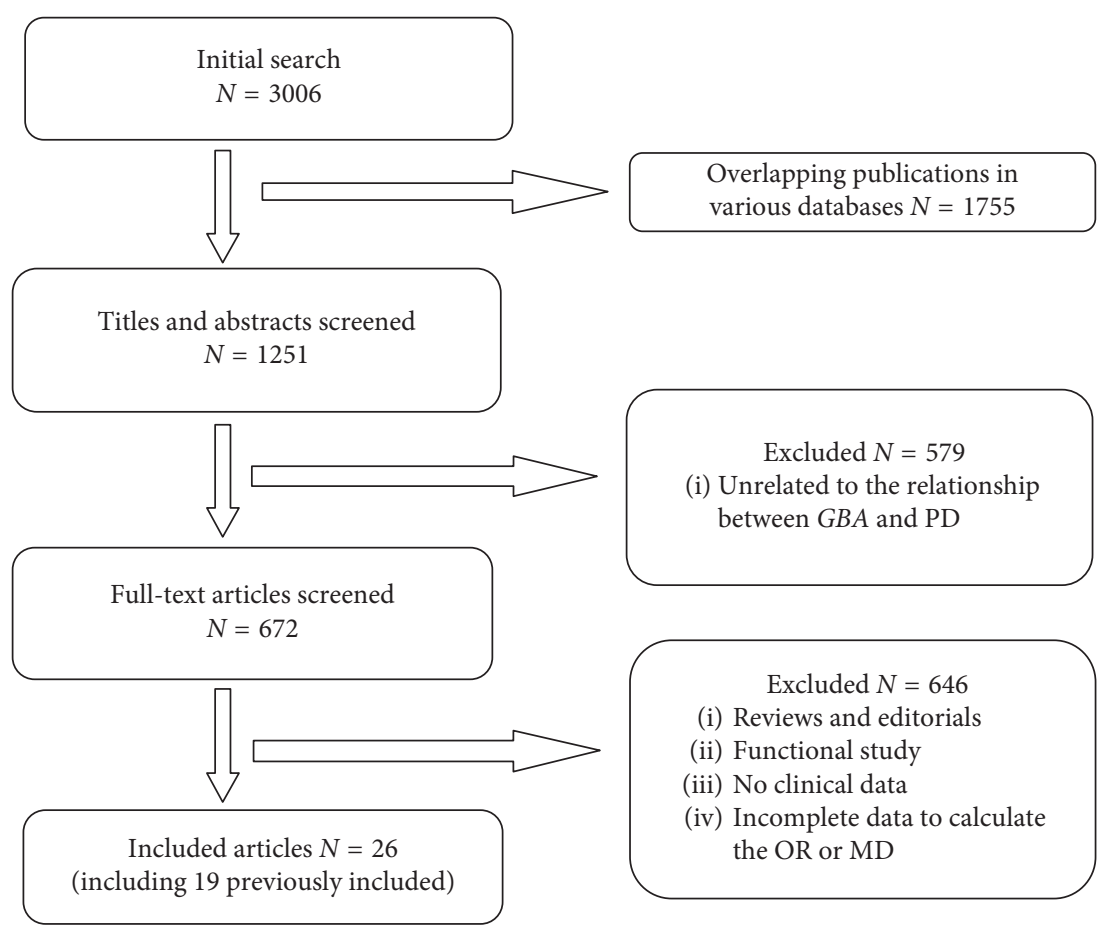

FIgURE 1: Flowchart illustrating the literature screening process.

in family history, AAO, UPDRS-III, and dementia (OR: 1.65; MD: $-3.61,2.17$; OR: 2.44; $p$ : $<0.00001,<0.00001,0.003$, and $<0.00001)$. We found a change in statistical differences in UPDRS-III scores from previous negative results, while we almost reached the same conclusion in analyzing family history, AAO, H-Y, and dementia.

All publications included were of high quality with the NOS scores above 7. According to the funnel plots (Supplementary Figure 2), the biases were rare. By removing articles one after another, the results of the remainder did not change significantly indicating that the results of our meta-analysis were stable.

\section{Discussion}

In our meta-analysis, we analyzed five new clinical features (depression, orthostatic hypotension, motor fluctuation, wearing-off, and freezing) and updated data of five previous analyzed clinical features (family history, AAO, UPDRSIII, H-Y, and dementia). We made the conclusion that $G B A+\mathrm{PD}$ patients had unique clinical features such as were more likely to have family history, earlier onset age, higher UPDRS-III scores, and develop dementia, depression, and wearing-off phenomena after adjusting disease duration.

Our meta-analysis about the demographic information of $G B A+\mathrm{PD}$ suggested that the carriers were more likely to have earlier age at onset with a mean of 3.6 years. Previous studies have shown that GBA + PD developed PD 1.7-6.0 years earlier than $G B A-\mathrm{PD}$ which were similar to our analysis [17]. Additionally, $G B A+\mathrm{PD}$ were more likely to have family history. These basic features of $G B A$ carriers will contribute to the targeted screening of the gene in researches.

As to other clinical features such as MSs and NMSs, our analysis demonstrated severe MSs reflected by higher UPDRS-III scores accompanied by motor complications like wearing-off phenomena and high possibilities to develop NMSs such as dementia and depression. Previous studies [18-20] have suggested deteriorative manifestations of $G B A+P D$ such as higher UPDRS-III scores, easily presenting dementia, and motor complications. Since separating different subcategories of PD is crucial to better understand disease mechanisms, predict disease progression, or design clinical researches, recently, Fereshtehnejad et al. [21] reported that important clinical features and scales such as UPDRS I-III scores, NMSs-related scales such as Montreal Cognitive Assessment (MoCA) evaluating cognitive functions, and Epworth Sleepiness Scale (ESS) evaluation sleep disturbances were key factors defining clinical subtypes of PD. Our analysis found unique clinical features of $G B A+P D$ which almost matched a diffuse malignant subtype in PD in the previous classifications which needed a more active treatment strategy for the deleterious prognosis.

The mechanism underlying $G B A+\mathrm{PD}$ prominent clinical features remains elusive. Some studies suggested that GBA mutations can cause dysfunctional GCase which finally led to $\alpha$-synuclein aggregations in PD brains and in dopaminergic neurons [22]. As $\alpha$-synuclein was vital pathological feature in $\mathrm{PD}$ brains, the promotion of $G B A$ mutations to $\alpha$-synuclein aggregations may explain the deleterious clinical features of $G B A+P D$. The dysfunctions in pathways outside classic basal ganglia may explain the NMS features of GBA+PD. The cortex dysfunction caused 


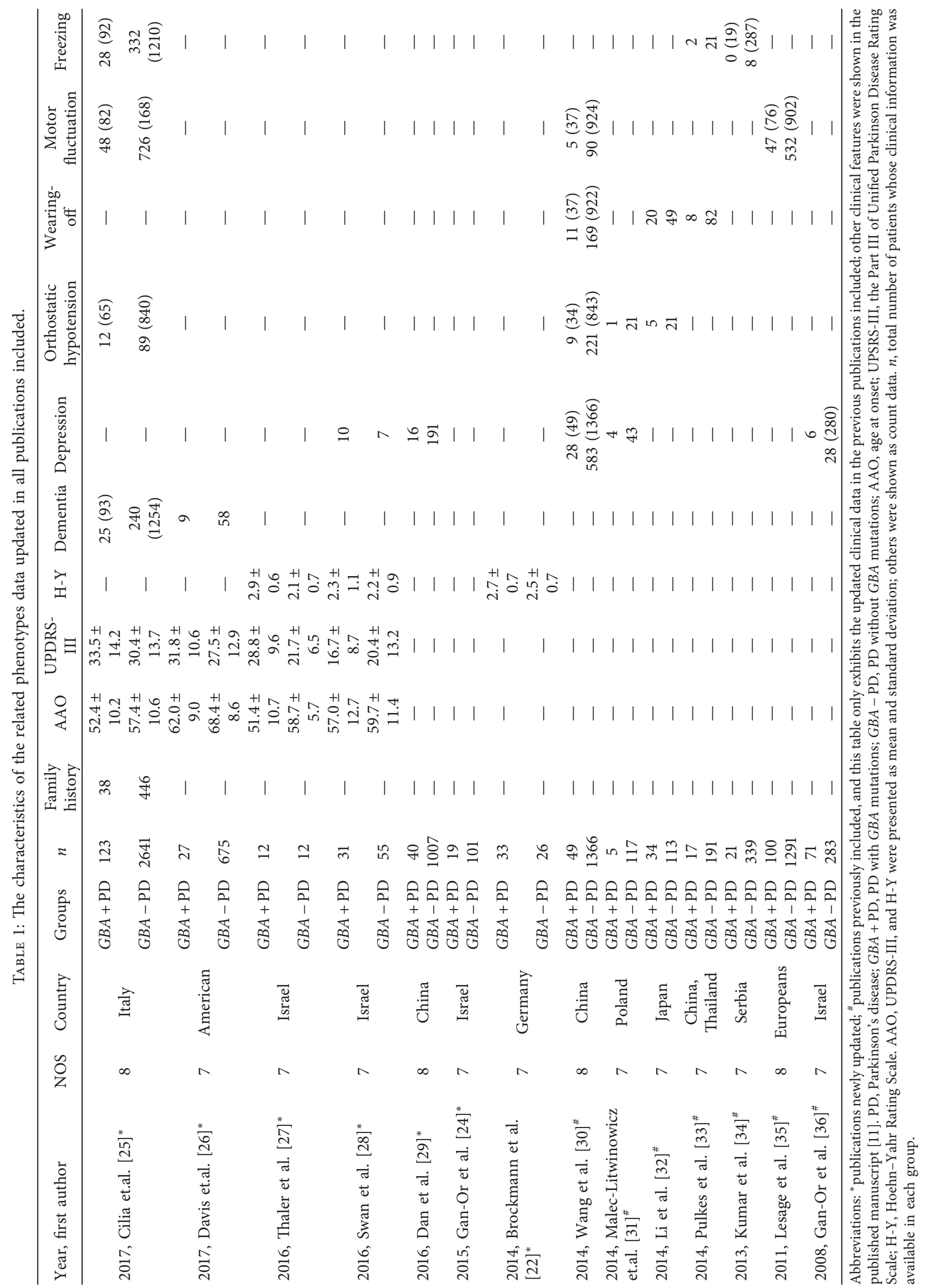


TABLE 2: GBA-related phenotypes updated to our previous meta-analysis.

\begin{tabular}{|c|c|c|c|c|c|c|}
\hline Phenotypes & $\begin{array}{l}\text { Number of articles } \\
\text { (total/updated) }\end{array}$ & $\begin{array}{c}\text { Total number of } \\
G B A+\mathrm{PD}\end{array}$ & $\begin{array}{c}\text { Total number of } \\
\text { GBA - PD }\end{array}$ & $\begin{array}{c}\text { OR or MD }(95 \% \mathrm{CI}) \\
\text { updated }\end{array}$ & $p$ value & $\begin{array}{c}\text { Previous OR or MD } \\
(95 \% \mathrm{CI})\end{array}$ \\
\hline Family history*a & $11 / 1$ & 558 & 9330 & $1.65(1.34,2.02)$ & $<0.00001$ & $1.5(1.18,1.91)$ \\
\hline $\mathrm{AAO}^{* \mathrm{~b}}$ & $17 / 4$ & 622 & 11079 & $\begin{array}{c}-3.61(-5.04 \\
-2.17)\end{array}$ & $<0.00001$ & $\begin{array}{c}-3.10(-4.88 \\
-1.32)\end{array}$ \\
\hline UPDRS-III*b & $9 / 4$ & 335 & 6100 & $2.17(0.72,3.62)$ & 0.003 & $1.61(-0.65,3.87)$ \\
\hline $\mathrm{H}-\mathrm{Y}^{* \mathrm{~b}}$ & $11 / 3$ & 275 & 3863 & $0.18(0.00,0.35)$ & 0.05 & $0.06(-0.06,0.17)$ \\
\hline Dementia*a & $8 / 2$ & 224 & 2696 & $2.44(1.79,3.33)$ & $<0.00001$ & $3.21(1.97,5.24)$ \\
\hline Depression $^{\# \mathrm{a}}$ & 5 & 196 & 2825 & $1.47(1.02,2.13)$ & 0.04 & - \\
\hline $\begin{array}{l}\text { Orthostatic } \\
\text { hypotension }^{\# a}\end{array}$ & 4 & 138 & 1913 & $1.24(0.79,1.94)$ & 0.35 & - \\
\hline $\begin{array}{l}\text { Motor } \\
\text { fluctuation }^{\# a}\end{array}$ & 3 & 195 & 2894 & $0.9(0.66,1.24)$ & 0.53 & - \\
\hline Wearing-off $\#$ a & 3 & 88 & 1226 & $1.68(1.05,2.69)$ & 0.03 & - \\
\hline Freezing $\#$ a & 3 & 128 & 1688 & $1.14(0.74,1.77)$ & 0.55 & - \\
\hline
\end{tabular}

Abbreviations: * phenotypes updated new publications; ${ }^{*}$ phenotypes newly analyzed. PD, Parkinson's disease; $G B A+\mathrm{PD}, \mathrm{PD}$ with $G B A$ mutations; GBA PD, PD without GBA mutations; AAO, age at onset; UPSRS-III, the Part III of Unified Parkinson Disease Rating Scale; OR, odds ratio; MD, mean deviation; CI, confidence interval. Bold OR or MD, $95 \% \mathrm{CI}$, and $p$ values reflected statistically significance results; a, dichotomous variables reflected by OR ( $95 \% \mathrm{CI})$; b, continuous outcomes reflected by MD (95\% CI).

by global brain degeneration can damage functions of specific areas of brains and cause dementia or depression [23]. However, the number of researches was limited, and more mechanism studies were needed in the future.

Previously, our comprehensive meta-analysis in GBA variants had proved the importance of $G B A$ mutations to $P D$ risks [10]. To clarify the role of GBA in PD clinical features more clearly, we did this meta-analysis. Our results demonstrated a clear phenotype-genotype correlation in $G B A$ + PD. Knowing the unique features of $G B A$ carriers will contribute to predicting the clinical course of $G B A+P D$ and be benefit for the symptomatic treatments. The results of this meta-analysis can do a contribution to the precise treatments based on genetic screening and help delay the progression of the disease with more active and effective therapeutic strategies.

To evaluate the meta-analysis more objectively, there were some limitations which cannot be ignored. First, possible biases were inevitable because the included original studies were cross-sectional and possible biases existed in pooled analyses of these studies such as age, gender, or other correlated clinical phenotypes. Further longitudinal designed studies will be needed to confirm these results. Second, because most of these researches included mixed different specific variants together as $G B A+\mathrm{PD}$ or $G B A-\mathrm{PD}$ (Supplementary Table 1 ), we could not separate each variant with corresponding phenotype data and were not able to conduct pooled analysis based on specific variants of GBA. With more original articles conducted based on specific variants of $G B A$ and phenotype, we may be able to do a more accurate analysis to help understand the relationship between the genotype and phenotype better. Third, although our updated meta-analysis was a comprehensive pooled analysis of $G B A$-associated clinical presentations, for the limited articles, we failed to prove the relationship of other clinical features such as rapid eye movement sleep behavior disorder (RBD) or freezing which were demonstrated to be associated with GBA previously [24].

\section{Conclusion}

Our meta-analysis suggested an increased risk of having family history, dementia, depression, wearing-off, earlier onset age, and higher UPDRS-III scores with GBA+PD. However, variants in $G B A$ had no relationship with $\mathrm{H}-\mathrm{Y}$, orthostatic hypotension, motor fluctuations, and freezing in PD. More data were needed to do complete analysis on different variants and different ethnics of $G B A$, and the corresponding clinical manifestations which can help the management of the whole duration of PD patients.

\section{Conflicts of Interest}

The authors declare that the research was conducted in the absence of any commercial or financial relationships that could be construed as potential conflicts of interest.

\section{Authors' Contributions}

YZ and LS have contributed equally to this work and are cofirst authors. YZ, LS, and QS chose the topic and designed the experiments. YZ, LS, and QS performed the analysis. YZ, LS, QS, and BT analyzed the data. YZ, LS, and QS wrote the manuscript. XZ, HP, QX, and JG performed data management and figure modification.

\section{Acknowledgments}

This work was supported by grants from the National Natural Science Foundation of China (Nos. 81430023 and 81401059), the National Key Plan for Scientific Research and Development of China (Nos. 2016YFC1306000 and 2017YFC0909100), and Hunan Provincial Innovation Foundation for Postgraduate (No. CX2017B066). 


\section{Supplementary Materials}

Supplementary Figure 1: forest plots of the association between phenotypes and $\mathrm{PD}$ risks in total. (A)-(J) respond to the phenotypes of family history, age at onset, UPDRS-III, $\mathrm{H}-\mathrm{Y}$, dementia, depression, orthostatic hypotension, motor fluctuation, wearing-off, and freezing individually. Supplementary Figure 2: funnel plots of the association between phenotypes and $\mathrm{PD}$ risks in total. $(\mathrm{A})-(\mathrm{J})$ respond to the phenotypes of family history, age at onset, UPDRS-III, H-Y, dementia, depression, orthostatic hypotension, motor fluctuation, wearing-off, and freezing individually. Supplementary Table 1: the GBA variants reported in included articles. Abbreviations: C, carriers of GBA variants; NC, noncarriers of GBA variants; NA, not available. (Supplementary Materials)

\section{References}

[1] L. V. Kalia and A. E. Lang, "Parkinson's disease," The Lancet, vol. 386, no. 9996, pp. 896-912, 2015.

[2] Y. Zhang, Q. Y. Sun, R. H. Yu, J.-f. Guo, B.-S. Tang, and X.-X. Yan, "The contribution of GIGYF2 to Parkinson's disease: a meta-analysis," Neurological Sciences, vol. 36, no. 11, pp. 2073-2079, 2015.

[3] K. Li, B. S. Tang, Z. H. Liu et al., "LRRK2 A419V variant is a risk factor for Parkinson's disease in Asian population," Neurobiology of Aging, vol. 36, no. 10, pp. 2908.e11-2908.e15, 2015.

[4] G. A. Grabowski, "Gaucher disease. enzymology, genetics, and treatment," Advances in Human Genetics 21, pp. 377-441, Springer Nature, Basel, Switzerland, 1993.

[5] Z. Yu, T. Wang, J. Xu et al., "Mutations in the glucocerebrosidase gene are responsible for Chinese patients with Parkinson's disease," Journal of Human Genetics, vol. 60, no. 2, pp. 85-90, 2015.

[6] A. Lwin, E. Orvisky, O. Goker-Alpan, M. E. LaMarca, and E. Sidransky, "Glucocerebrosidase mutations in subjects with parkinsonism," Molecular Genetics and Metabolism, vol. 81, no. 1, pp. 70-73, 2004.

[7] G. O’Regan, R. M. deSouza, R. Balestrino, and A. H. Schapira, "Glucocerebrosidase mutations in Parkinson disease," Journal of Parkinson's Disease, vol. 7, no. 3, pp. 411-422, 2017.

[8] K. Fan, B. S. Tang, Y. Q. Wang et al., "The GBA, DYRK1A and MS4A6A polymorphisms influence the age at onset of Chinese Parkinson patients," Neuroscience Letters, vol. 621, pp. 133-136, 2016.

[9] J. F. Guo, K. Li, R. L. Yu et al., "Polygenic determinants of Parkinson's disease in a Chinese population," Neurobiology of Aging, vol. 36, no. 4, pp. 1765.e1-1765.e6, 2015.

[10] Y. Zhang, L. Shu, Q. Sun et al., "Integrated genetic analysis of racial differences of common GBA variants in Parkinson's disease: a meta-analysis," Frontiers in Molecular Neuroscience, vol. 11, 2018.

[11] Y. Zhang, Q. Y. Sun, Y. W. Zhao et al., "Effect of GBA mutations on phenotype of Parkinson's disease: a study on Chinese population and a meta-analysis," Parkinson's Disease, vol. 2015, article 916971, 10 pages, 2015.

[12] J. Aharon-Peretz, H. Rosenbaum, and R. Gershoni-Baruch, "Mutations in the glucocerebrosidase gene and Parkinson's disease in Ashkenazi Jews," New England Journal of Medicine, vol. 351, no. 19, pp. 1972-1977, 2004.
[13] C. P. da Silva, M. A. G. de, P. H. Cabello Acero et al., "Clinical profiles associated with LRRK2 and GBA mutations in Brazilians with Parkinson's disease," Journal of the Neurological Sciences, vol. 381, pp. 160-164, 2017.

[14] R. Torok, D. Zadori, N. Torok et al., "An assessment of the frequency of mutations in the GBA and VPS35 genes in Hungarian patients with sporadic Parkinson's disease," Neuroscience Letters, vol. 610, pp. 135-138, 2016.

[15] A. J. Hughes, S. E. Daniel, L. Kilford, and A. J. Lees, "Accuracy of clinical diagnosis of idiopathic Parkinson's disease: a clinico-pathological study of 100 cases," Journal of Neurology, Neurosurgery \& Psychiatry, vol. 55, no. 3, pp. 181-184, 1992.

[16] A. Stang, "Critical evaluation of the Newcastle-Ottawa scale for the assessment of the quality of nonrandomized studies in meta-analyses," European Journal of Epidemiology, vol. 25, no. 9, pp. 603-605, 2010.

[17] R. Asselta, V. Rimoldi, C. Siri et al., "Glucocerebrosidase mutations in primary parkinsonism," Parkinsonism \& Related Disorders, vol. 20, no. 11, pp. 1215-1220, 2014.

[18] M. Beavan, A. McNeill, C. Proukakis et al., "Evolution of prodromal clinical markers of Parkinson disease in a GBA mutation-positive cohort," JAMA Neurology, vol. 72, no. 2, pp. 201-208, 2015.

[19] T. Oeda, A. Umemura, Y. Mori et al., "Impact of glucocerebrosidase mutations on motor and nonmotor complications in Parkinson's disease," Neurobiology of Aging, vol. 36, no. 12, pp. 3306-3313, 2015.

[20] B. Creese, E. Bell, I. Johar, P. Francis, C. Ballard, and D. Aarsland, "Glucocerebrosidase mutations and neuropsychiatric phenotypes in Parkinson's disease and lewy body dementias: review and meta-analyses," American Journal of Medical Genetics Part B: Neuropsychiatric Genetics, vol. 177, no. 2, pp. 232-241, 2018.

[21] S. M. Fereshtehnejad, Y. Zeighami, A. Dagher, and R. B. Postuma, "Clinical criteria for subtyping Parkinson's disease: biomarkers and longitudinal progression," Brain, vol. 140, no. 7, pp. 1959-1976, 2017.

[22] K. Brockmann, K. Srulijes, S. Pflederer et al., "GBA-associated Parkinson's disease: reduced survival and more rapid progression in a prospective longitudinal study," Movement Disorders, vol. 30, no. 3, pp. 407-411, 2015.

[23] M. Beavan and A. H. Schapira, "Glucocerebrosidase gene mutation and preclinical markers of Parkinson disease-reply," JAMA Neurology, vol. 72, no. 6, p. 724, 2015.

[24] Z. Gan-Or, A. Mirelman, R. B. Postuma et al., "GBA mutations are associated with rapid eye movement sleep behavior disorder," Annals of Clinical and Translational Neurology, vol. 2, no. 9, pp. 941-945, 2015.

[25] R. Cilia, S. Tunesi, G. Marotta et al., "Survival and dementia in GBA-associated Parkinson's disease: the mutation matters," Annals of Neurology, vol. 80, no. 5, pp. 662-673, 2016.

[26] M. Y. Davis, C. O. Johnson, J. B. Leverenz et al., “Association of GBA mutations and the E326K polymorphism with motor and cognitive progression in Parkinson disease," JAMA Neurology, vol. 73, no. 10, pp. 1217-1224, 2016.

[27] A. Thaler, T. Gurevich, A. Bar Shira et al., "A "dose" effect of mutations in the GBA gene on Parkinson's disease phenotype," Parkinsonism \& Related Disorders, vol. 36, pp. 47-51, 2017.

[28] M. Swan, N. Doan, R. A. Ortega et al., "Neuropsychiatric characteristics of GBA-associated Parkinson disease," Journal of the Neurological Sciences, vol. 370, pp. 63-69, 2016.

[29] X. Dan, C. Wang, J. Zhang et al., "Association between common genetic risk variants and depression in Parkinson's 
disease: A dPD study in Chinese," Parkinsonism \& Related Disorders, vol. 33, pp. 122-126, 2016.

[30] C. Wang, Y. Cai, Z. Gu et al., "Clinical profiles of Parkinson's disease associated with common leucine-rich repeat kinase 2 and glucocerebrosidase genetic variants in Chinese individuals," Neurobiology of Aging, vol. 35, no. 3, pp. 725.e1-725.e6, 2014.

[31] M. Malec-Litwinowicz, M. Rudzinska, M. Szubiga, M. Michalski, T. Tomaszewski, and A. Szczudlik, "Cognitive impairment in carriers of glucocerebrosidase gene mutation in Parkinson disease patients," Neurologia i Neurochirurgia Polska, vol. 48, no. 4, pp. 258-261, 2014.

[32] Y. Li, T. Sekine, M. Funayama et al., "Clinicogenetic study of GBA mutations in patients with familial Parkinson's disease," Neurobiology of Aging, vol. 35, no. 4, pp. 935.e3-935.e8, 2014.

[33] T. Pulkes, L. Choubtum, S. Chitphuk et al., "Glucocerebrosidase mutations in Thai patients with Parkinson's disease," Parkinsonism \& Related Disorders, vol. 20, no. 9, pp. 986-991, 2014.

[34] K. R. Kumar, A. Ramirez, A. Gobel et al., "Glucocerebrosidase mutations in a Serbian Parkinson's disease population," European Journal of Neurology, vol. 20, no. 2, pp. 402-405, 2013.

[35] S. Lesage, M. Anheim, C. Condroyer et al., "Large-scale screening of the Gaucher's disease-related glucocerebrosidase gene in Europeans with Parkinson's disease," $\mathrm{Hu}$ man Molecular Genetics, vol. 20, no. 1, pp. 202-210, 2011.

[36] Z. Gan-Or, N. Giladi, U. Rozovski et al., "Genotypephenotype correlations between GBA mutations and Parkinson disease risk and onset," Neurology, vol. 70, no. 24, pp. 2277-2283, 2008. 


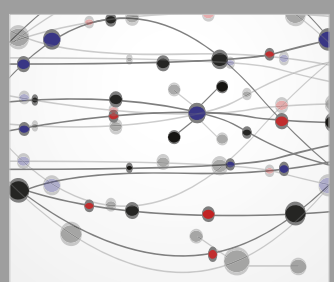

The Scientific World Journal
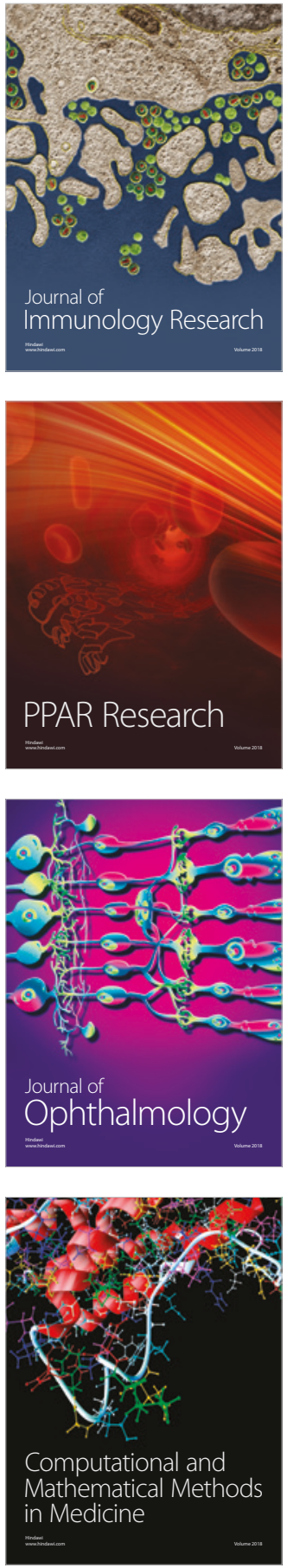

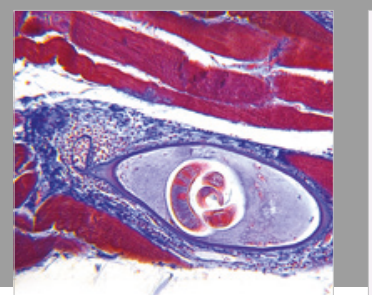

Gastroenterology Research and Practice

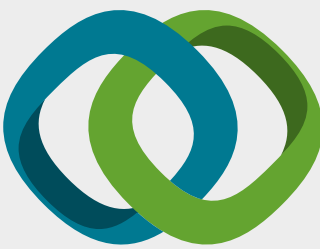

\section{Hindawi}

Submit your manuscripts at

www.hindawi.com
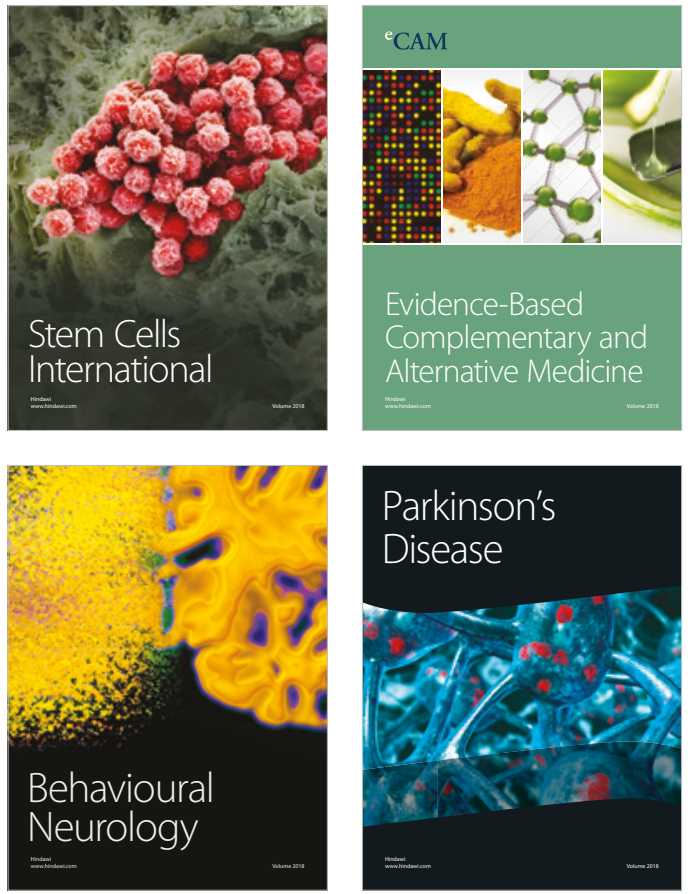

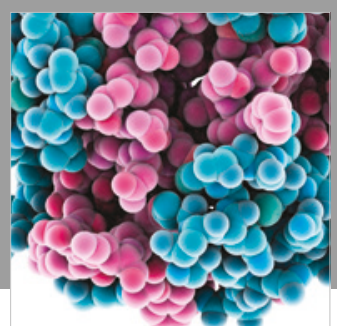

ournal of

Diabetes Research

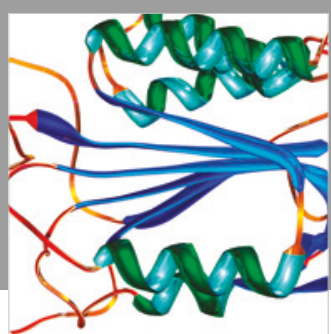

Disease Markers
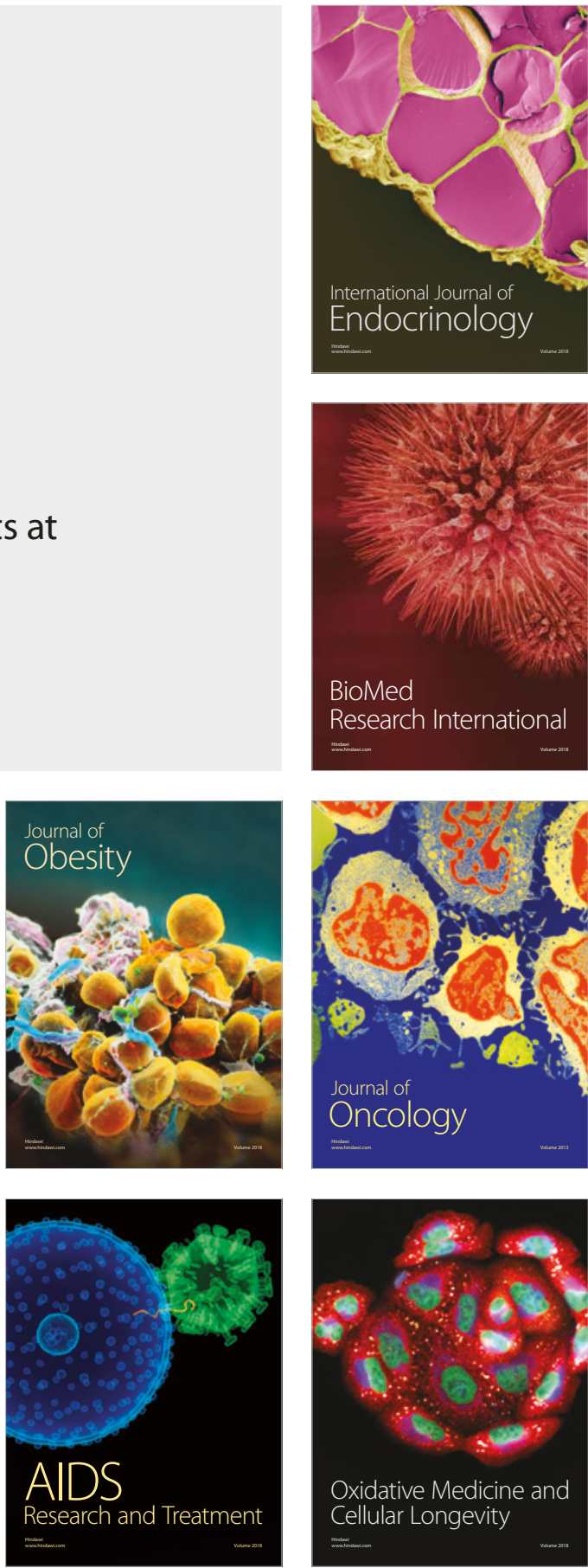